\title{
Correction
}

\section{Correction: Albert and Shadmehr, "The Neural Feedback Response to Error As a Teaching Signal for the Motor Learning System"}

In the article "The Neural Feedback Response to Error As a Teaching Signal for the Motor Learning System” by Scott T. Albert and Reza Shadmehr, which appeared on pages 4832-4845 of the April 27, 2016 issue, the authors wish to correct an issue with the purple perpendicular displacement traces in Figure 2A, labeled "Error 2". In the original figure, these traces mistakenly show the average error to the first and second perturbations of quartet trial progressions, rather than solely the second error as indicated by the figure caption. In conjunction with this issue, the authors would like to report that the statistically significant reduction in peak displacement from Error 1 to Error 2 reported on pg. 4837 is still correct, though the corresponding $p$ values are in fact $p<10^{-28}$ (CW perturbations) and $p<10^{-26}$ (CCW perturbations) as opposed to $p<10^{-23}$ which is reported in the original article. This correction is in line with the trend already reported in the original manuscript and therefore does not affect any of the results, interpretations, or conclusions of the article. All of the electromyographic data in Figure 2B are unaffected by this error, and all other figures in the original article remain correct. The corrected Figure 2 is shown below and has also been corrected on the online PDF version.

DOI: 10.1523/JNEUROSCI.2857-16.2016 
A

Clockwise perturbation

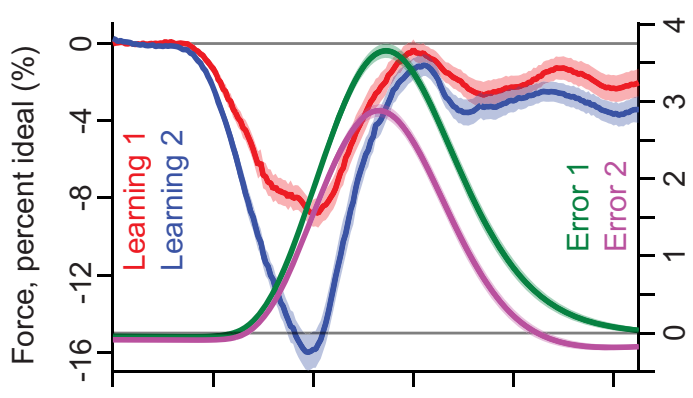

B
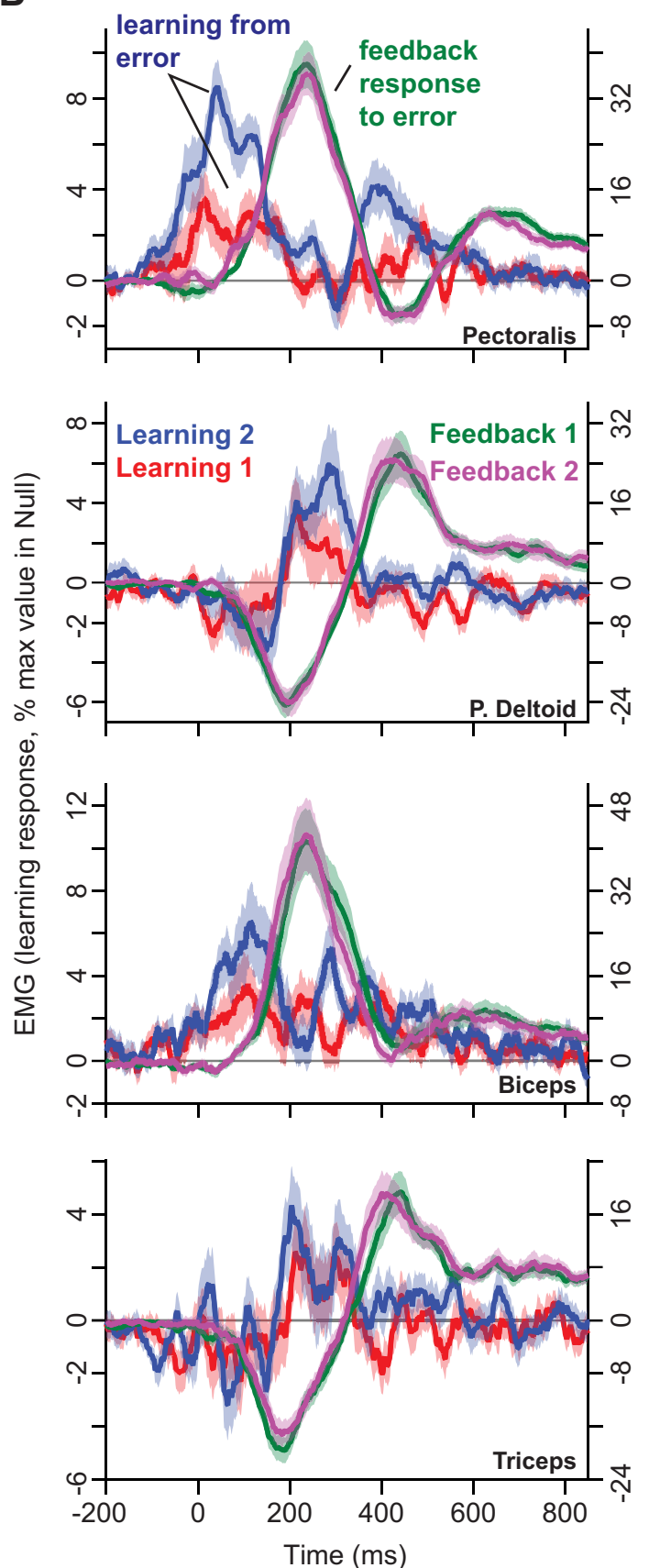

Counter-clockwise perturbation

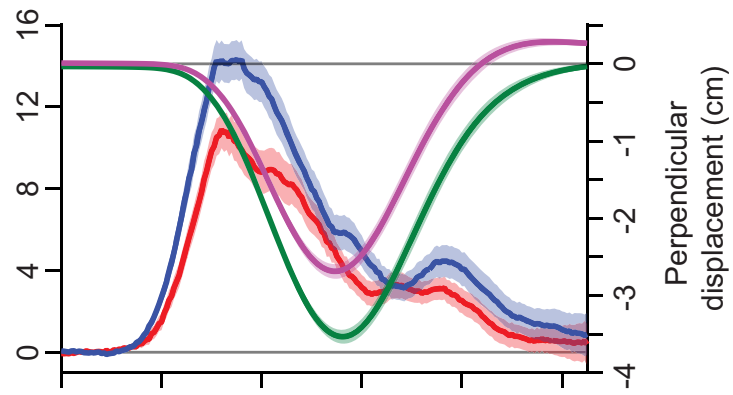

feedback response
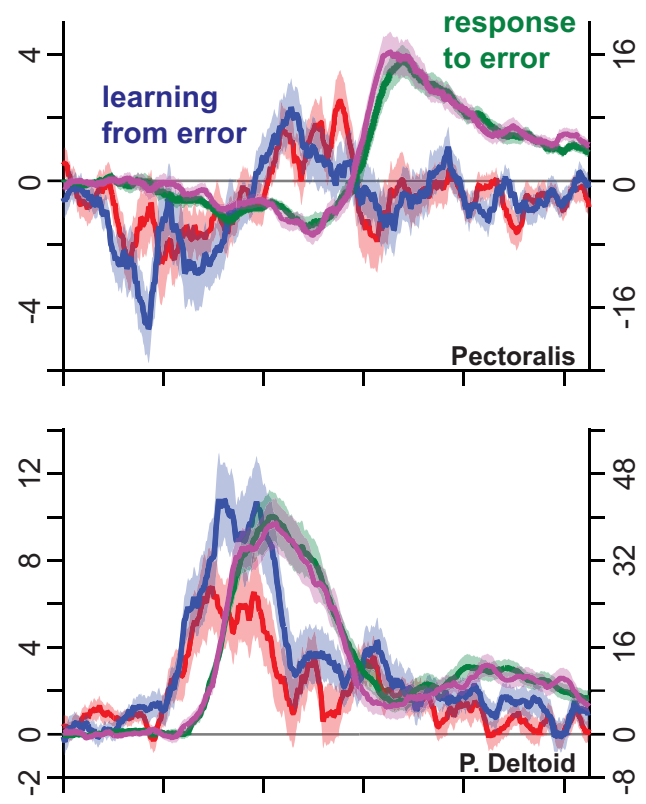

+

กิ

$\because \frac{0}{\frac{1}{2}}$

x
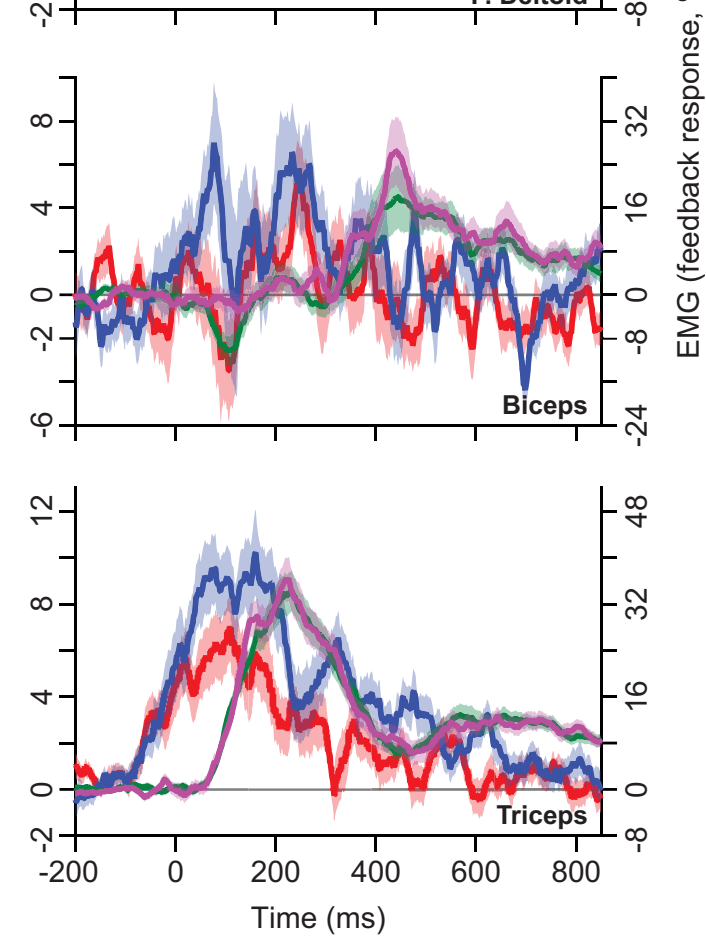

Figure 2. 